\title{
Psychometric validation of the Japanese version of Alcohol Quality of Life Scale (AQoLS-Japan) in the treatment of patients with alcohol use disorder
}

\author{
Susumu Higuchi ${ }^{1} \cdot$ Yoshiya Moriguchi $^{2}$ (1) $\cdot$ Kristin Hui Xian $\operatorname{Tan}^{3}$
}

Accepted: 19 September 2019 / Published online: 4 October 2019

(c) The Author(s) 2019

\begin{abstract}
Purpose The Alcohol Quality of Life Scale (AQoLS) is accepted as a useful measure in assessing impact of alcohol use disorders (AUD) on health-related quality of life (HR-QoL) in Western cultures. We aimed to assess the psychometric properties of the Japanese version of the AQoLS (AQoLS-Japan).

Methods This was a 3-month, observational cohort study in patients undergoing routine treatment for AUD in Japan. HRQoL was assessed using the AQoLS-Japan (34 items, 7 dimensions). Scale psychometrics were analyzed using correlative techniques.

Results Data from 132 patients were analyzed. Inter-item and item-scale correlations for the AQoLS-Japan scale were moderate to strong. Confirmatory factor analysis results supported the AQoLS-Japan structure but there was evidence of interdependency among some items and factors. Cronbach's alpha coefficients for internal consistency ranged from 0.73 to 0.97, and intraclass correlation coefficients for scores between test (baseline) and retest ( 2 weeks) ranged from 0.65 to 0.82. Convergent and divergent validity and known-groups validity were supported. Evaluation of within-group change demonstrated that the AQoLS-Japan total and domains consistently demonstrated statistically significant improvement $(p<0.001$ in all cases) in HR-QoL over time. Estimates for minimal clinically important difference on the AQoLS-Japan total score ranged from 13.2 to 18.2 for group-level change and from 2.4 to 15.7 for a group-level difference.

Conclusions The AQoLS-Japan is a reliable and valid measure of HR-QoL that is able to demonstrate benefits associated with the routine treatment of AUD in Japan.
\end{abstract}

Keywords Alcohol use disorders · Quality of life · Japan

\section{Introduction}

Alcohol use disorders (AUD), including alcohol abuse and dependence, pose a great health, social and economic burden to affected individuals, their families, and society. The

Electronic supplementary material The online version of this article (https://doi.org/10.1007/s11136-019-02310-w) contains supplementary material, which is available to authorized users.

Yoshiya Moriguchi

YOMO@lundbeck.com

1 National Hospital Organization Kurihama Medical and Addiction Center, 5-3-1 Nobi, Yokosuka, Kanagawa 239-0841, Japan

2 Lundbeck Japan K.K, Tokyo, Japan

3 Lundbeck Singapore Pte Ltd, Singapore, Singapore harmful use of alcohol is one of the world's leading health risks [1], and the greater the volume of alcohol that an individual consumes, the greater the risks associated with its use. In 2018, the World Health Organization (WHO) estimated that $22.8 \%$ of the general population (aged $15+$ years) participate in heavy episodic drinking, and that $3.4 \%$ of the Japanese population have an AUD (12-month prevalence estimate) [2]. However, various surveys have reported that the majority (up to 70\%) of Japanese heavy drinkers are unaware that their drinking behavior may be problematic [3] and only about 5\% of alcohol-dependent persons in Japan actually seek medical advice and treatment [4]. It has been suggested that this lack of awareness is, at least partly, attributable to Japanese drinking culture, where there is high tolerance for drinking alcohol to facilitate socialization [5]. Conversely, there is significant stigma associated with alcoholism in Japan, where people with AUDs are often 
stereotyped as having 'weak will' and socially unacceptable which likely deters them from seeking treatment [6].

In order to be able to assess the effectiveness of potential alcohol reduction strategies in a 'people first' approach, the field has started to move away from traditional measures of drinking (e.g., total alcohol consumption [TAC], or number of heavy drinking days [HDD]) to additional outcomes that are closer to patient needs. Health-related quality of life (HR-QoL) is acknowledged as critical domain to consider when assessing the effectiveness of treatment for AUDs and is often included as a secondary endpoint in clinical trials [7, 8]. However, a 2012 systematic review of AUD studies using generic HR-QoL scales found that they were not well suited to showing treatment effects in subjects with AUD [9]. Thus, the Alcohol Quality of Life Scale (AQoLS) was developed as the first AUD-specific measure of HR-QoL for increased sensitivity in showing the effectiveness of therapeutic interventions from the patient's perspective [10].

Scale psychometrics of the French and English (UK) versions of the AQoLS have been evaluated and have demonstrated construct validity and internal consistency [11]. The development process for the original scale also included a formal translatability assessment by translation experts to ensure the items were amenable to translation and cultural adaptation. The original English (UK) version of AQoLS was translated into Japanese, and formal cultural adaptation was completed in July 2013 [9]. We present here the first assessment of the psychometric properties of the Japanese version of the Alcohol Quality of Life Scale (AQoLS-Japan) in Japanese patients with an AUD.

\section{Methods}

This was a multi-site, observational, prospective, longitudinal, cohort study in patients with AUD, followed in specialized care for up to 3 months ( \pm 2 weeks) in Japan. Patients were treated according to routine practice, i.e., treatment was not decided in advance by the study protocol. The study was conducted between October 4, 2016 and September 5, 2017, at 15 outpatient sites across Japan.

\section{Patients}

Patients were male and female Japanese adults (aged $\geq 20$ years) with a diagnosis of AUD according to the Diagnostic and Statistical Manual of Mental Disorders, 5th Edition (DSM-5). Eligible patients had to be currently on an established outpatient treatment plan with the intent to follow that plan for 3 months or have an established treatment plan that started within 1-4 weeks after the baseline visit. Key exclusion criteria were any serious or unstable psychiatric disorders (such as drug addiction) and physical disorders that prevented participation into the study, learning difficulties that prevented him/her reading and understanding questionnaires (e.g., dementia), and, in the physician's opinion, could not be followed for the whole duration of the study.

\section{Assessments}

Assessments were conducted as part of routine practice visits at baseline, 2 weeks ( \pm 1 week), and 3 months ( \pm 2 weeks). Patients self-rated their HR-QoL using the AQoLS-Japan which has 34 items across 7 dimensions: activities (items $2-7,13,15$, and 25-26), relationships (items 1, 8-11, and 27 ), living conditions (items $16-18$ and 24 ), negative emotions (items 22-23), control (items 28-32), sleep (items 33-34), and self-esteem (items 12, 14, and 19-21) (Supplementary Appendix Table e1). Each item has four response categories (not at all, a little, quite a lot, and very much), with a 4-week recall period. Dimension and total (sum of 34 items) scores are linearly transformed to a 0-100 range, with higher scores indicating poorer HR-QoL. Other HRQoL assessments included Japanese versions of generic HR-QoL measures - the EuroQol questionnaire (EQ-5D-3L) [12] and the SF-36 Health Survey (version 2) (SF-36) [13]. Clinicians and patients rated their global impressions of severity using the 7-item Clinical Global Impression of Severity (CGI-S) and the 5-item Patient Global Impression of Severity (PGI-S), respectively. Impressions of change were assessed using the Clinical Global Impression of Improvement (CGI-I) and Patient Global Impression of Change (PGI-C) [14], both consisting of 7 items where $1=$ very much improved/better, $2=$ much improved/better, $3=$ minimally improved/better, $4=$ no change, $5=$ minimally worse, $6=$ much worse, and $7=$ very much worse). Levels of alcohol consumption were evaluated using the Timeline Follow-Back method (past 28 days) [15]. Drinking risk levels (DRLs) were defined according to WHO criteria as [male/female] low $\leq 40 \mathrm{~g} / \leq 20 \mathrm{~g}$; medium 41-60 g/21-40 g; high $61-100 \mathrm{~g} / 41-60 \mathrm{~g}$; and very high $>100 \mathrm{~g} />60 \mathrm{~g}$ per day) [2]. A DRL response was defined as from very high to medium/low DRL, high to low DRL, medium to low DRL, or low DRL to alcohol consumption of $0 \mathrm{~g} /$ day.

\section{Statistical analyses}

\section{Sample size estimation}

The target sample size was determined based on the requirement to have approximately 60 patients for the assessment of test-retest reliability and responsiveness [16]. Based on the results of two previous clinical trials in patients with AUD $[17,18]$, it was estimated that approximately $59 \%$ of patients would maintain their baseline DRL after 2 weeks 
of follow-up and $58 \%$ would achieve treatment response at 3 months. Hence, considering 18\% withdrawal rate at 3 months, it was estimated that a minimum of 127 patients were needed for this study. To allow for any cultural differences between Japan and the countries involved in the clinical trials (e.g., higher loss to follow-up), the minimum enrollment was increased to 150 patients.

\section{Analysis population}

The analysis population included all patients who met selection criteria and completed the baseline assessment. Psychometric validation assessments were performed for all patients who completed all study visits.

\section{Descriptive statistics}

Standard descriptive statistics were used to describe the distributional properties of the AQoLS-Japan item, dimension, and total scores at each study visit, as well as for change from baseline for AQoLS-Japan dimension and total scores at follow-up visits.

Descriptive statistics were also used to summarize the HR-QoL and clinical status of patients with AUD (AQoLSJapan, EQ-5D, SF-36, PGI-S, and PGI-C) at baseline and 3 months.

\section{Psychometric validation}

Dimensional structure was assessed through inter-item and item-scale correlations at each visit and confirmatory factor analysis (CFA) using data from baseline. Adequacy of model fit was evaluated through the model Chi-square test statistic, comparative fit index (CFI $\geq 0.95)$, Tucker-Lewis index ( $T L I \geq 0.95$ ), and the root mean square error of approximation (RMSEA $\leq 0.06$ ). Internal reliability of AQoLS-Japan was evaluated through Cronbach's alpha coefficients, where an alpha between 0.70 and 0.90 indicates a set of items that is strongly related but not redundant and that is capable of supporting a unidimensional scoring structure [19].

Test-retest reliability was evaluated in patients whose condition remained stable on PGI-C, PGI-S, CGI-I, and DRL between baseline (test) and 2 weeks (retest) by estimating the intraclass correlation coefficients (ICCs) for scores between test and retest; ICCs of $\geq 0.70$ were taken to represent adequate reliability. Construct validity was investigated by testing a priori hypotheses to evaluate the direction and strength of the relationships between AQoLS-Japan scores and scores on comparator measures (SF-36, EQ-5D, PGI-S, CGI-S, and alcohol consumption) using Pearson productmoment correlations at each visit. The strength of correlations is assessed based on Cohen's criteria [20]; correlations between 0.10 and 0.29 are considered small, correlations between 0.30 and 0.49 are considered moderate, and correlations of 0.50 or greater are considered strong. A priori hypotheses regarding the direction and strength of these correlations were determined based on the literature and findings from the UK and French AQoLS psychometric validation $[8,11]$. We hypothesized that there would be:

1. Moderate to strong negative correlation between the AQoLS and the SF-36 mental and role-social components.

2. Moderate negative correlations between the AQoLS and the SF-36 role emotional, vitality, mental health, and social functioning components.

3. Small to moderate negative correlation between the AQoLS and the EQ-5D visual analogue scale.

4. Low to moderate positive correlation between the AQoLS and the CGI-S; stronger correlation between the AQoLS and the PGI-S.

5. Low to moderate positive correlations between the AQoLS and measures of alcohol consumption.

Since there is a well-established, 'dose-related' continuum of health impact for AUD [21], we wanted to check scale validity across the spectrum of drinking behaviors, from mild through to more severe disease and at different levels of alcohol consumption. Known-groups validity was evaluated through statistical significance of differences in AQoLS-Japan scores between the two most extreme subgroups across known prespecified subgroups (disease severity based on PGI-S and CGI-S, and level of consumption based on number of HDDs, number of drinking days and DRL) using t tests at each visit.

The ability to detect change of the AQoLS-Japan was evaluated by using Pearson product-moment correlation coefficients between AQoLS-Japan Month-3 change from baseline and Month-3 change on comparator measures (SF-36, EQ-5D, alcohol consumption, PGI-C, and CGII). AQoLS-Japan change in scores between baseline and 3 months were also computed for patients who improved on PGI-C, CGI-I, and reduced alcohol consumption during the 3-month follow-up period and the significance of change was tested using paired $t$ test. In addition, effect-size estimate of change and standardized response mean were computed for the mean change in scores between baseline and 3 months on the AQoLS dimensions and total. Responsiveness effect sizes were interpreted based on [22] guidelines [22], where $\geq 0.20$ to $<0.50$ represent small effects, $\geq 0.50$ to $<0.80$ represent moderate effects, and $\geq 0.80$ represent large effects. The minimal clinically important difference (MCID) is defined as the smallest change or difference in scores of a measure perceived by patients as beneficial or harmful [23]. Estimates for an MCID to evaluate group-level change over time on the AQoLS total were determined using 
the AQoLS total mean change for patients who had a small improvement (defined as PGI-S $=1$, PGI-C $=3$, CGI-S $=1$ or 2, CGI-I $=3$, or DRL improvement of one category) between baseline and Month 3 on each anchor determined to be adequate [23-25]. Estimates for responder definitions were based on mean changes, receiver-operator characteristic (ROC) analysis, and cumulative distribution function plots.

All statistical tests were two-sided and conducted at the $5 \%$ level of significance. With the exception of the SF-36 (where missing data at the item level were treated in accordance with standard scoring guidelines [13]), imputation was not performed for missing data. The statistical software used was $\mathrm{SAS}^{\circledR}$, Version 9.4.

\section{Results}

\section{Patient disposition and baseline characteristics}

A total sample of 150 patients completed baseline assessments and were included in the study. Of these, five patients were lost to follow-up between baseline and the Week-2 visit and a further 12 were lost to follow-up between Week 2 and Month 3. Data from 132 of the 133 patients who completed all study visits were used for psychometric validation as one patient did not complete the AQoLS-Japan at Month-3 visit.

Table 1 Baseline characteristics

\begin{tabular}{|c|c|c|}
\hline & Overall $(N=150)$ & $\begin{array}{l}\text { Psychometric } \\
\text { analysis group } \\
(N=132)\end{array}$ \\
\hline \multicolumn{3}{|l|}{ Sex; $n(\%)$} \\
\hline Male & $123(82.0 \%)$ & $108(81.8 \%)$ \\
\hline Female & $27(18.0 \%)$ & $24(18.2 \%)$ \\
\hline Age (years); mean (SD) & $52.9(12.1)$ & $53.2(12.4)$ \\
\hline \multicolumn{3}{|l|}{ Living status; $n(\%)$} \\
\hline Alone & $58(38.7 \%)$ & $51(38.6 \%)$ \\
\hline Not alone & $92(61.3 \%)$ & $81(61.4 \%)$ \\
\hline \multicolumn{3}{|l|}{ Employment status; $n(\%)$} \\
\hline On a job & $62(41.3 \%)$ & $51(38.6 \%)$ \\
\hline Working at home & $6(4.0 \%)$ & $6(4.5 \%)$ \\
\hline Retired & $18(12.0 \%)$ & $17(12.9 \%)$ \\
\hline No job & $64(42.7 \%)$ & $58(43.9 \%)$ \\
\hline \multicolumn{3}{|l|}{ Marital status; $n(\%)$} \\
\hline Not married & $36(24.0 \%)$ & $32(24.2 \%)$ \\
\hline Married/living with someone & $65(43.3 \%)$ & $54(40.9 \%)$ \\
\hline Divorced/separated & $45(30.0 \%)$ & $42(31.8 \%)$ \\
\hline Bereavement & $4(2.7 \%)$ & $4(3.0 \%)$ \\
\hline Current smoker; $n(\%)$ & $100(67.1 \%)$ & $90(68.7 \%)$ \\
\hline \multicolumn{3}{|l|}{ Alcohol use disorder; $n(\%)$} \\
\hline Abuse & $4(2.7 \%)$ & $3(2.3 \%)$ \\
\hline Dependence & $146(97.3 \%)$ & $129(97.7 \%)$ \\
\hline Total alcohol consumption (g/day) over 28 days; mean (SD) & $37.1(48.1)$ & $33.3(43.9)$ \\
\hline Number of drinking days; mean (SD) & $11.8(11.1)$ & $10.8(10.9)$ \\
\hline Number of heavy drinking days; mean (SD) & $8.5(10.4)$ & $7.4(9.8)$ \\
\hline Age at onset of drinking problem (years); mean (SD) & $39.0(13.7)$ & $38.9(13.7)$ \\
\hline Time since diagnosis (years); mean (SD) & $3.8(5.4)$ & $4.1(5.5)$ \\
\hline $\begin{array}{l}\text { Number of previous attempts to abstain or reduce alcohol } \\
\text { consumption; mean (SD) }\end{array}$ & $9.0(20.1)$ & $9.8(21.3)$ \\
\hline \multicolumn{3}{|l|}{ Comorbidities; $n(\%)$} \\
\hline Cardiovascular & $33(22.0 \%)$ & $30(22.7 \%)$ \\
\hline Gastric & $28(18.7 \%)$ & $23(17.4 \%)$ \\
\hline Hepatic & $64(42.7 \%)$ & $54(40.9 \%)$ \\
\hline Metabolic & $27(18.0 \%)$ & $22(16.7 \%)$ \\
\hline Psychiatric & $55(36.7 \%)$ & $48(36.4 \%)$ \\
\hline
\end{tabular}


Most patients $(82.0 \%)$ were male and the average age was 52.9 years. Baseline demographic and clinical characteristics for patients are shown in Table 1 and were similar between the total sample of 150 patients and the 132 patients with AQoLS-Japan data considered for psychometric validation. The most common therapeutic goal was to abstain completely from alcohol (89.9\%). Most enrolled patients $(60.0 \%)$ were continuing on their current treatment plan (average of 4.7 years since initiation of treatment plan), with the rest initiating their plan at the start of the study. In line with routine care in Japan, all patients received some form of non-pharmacologic therapy (brief advice $41.1 \%$, individual therapy $93.0 \%$, group therapy $43.4 \%$, education program $41.1 \%$, and family intervention $13.2 \%$ ). Over half $(58.9 \%)$ of patients received pharmacologic treatment at baseline (38.8\% received acamprosate and $13.2 \%$ received disulfiram).

\section{Treatment effect}

Reductions in alcohol consumption with routine treatment were observed at 2 weeks and 3 months. Mean TAC was reduced from a mean of $37.1 \mathrm{~g} /$ day at baseline to $15.0 \mathrm{~g} /$ day at 2 weeks and to $11.8 \mathrm{~g} /$ day at 3 months. The number of drinking days reduced from a mean of 11.8 drinking days (42.0\% of days drinking) at baseline to 4.0 drinking days (14.3\% of days drinking) at 3 months. Overall $57.1 \%$ (56/98) patients showed a DRL response by 3 months.

Reductions in drinking measures were reflected in patient and clinician impressions of change and in HRQoL. According to the PGI-C, $71.1 \%$ of study patients improved at 2 weeks and $80.2 \%$ improved at 3 months. According to CGI-I, $58.4 \%$ of study patients improved at 2 weeks and $58.6 \%$ improved at 3 months. The mean \pm SD AQoLS-Japan total score was $30.4 \pm 22.5$ at baseline reducing to $16.8 \pm 18.4$ at 3 months, indicating an improvement (Table 2). Patients also showed improvement in generic measures of HR-QoL. Mean EQ-5D visual analogue scale scores improved from 68.5 at baseline to 75.3 at 3 months. In terms of the SF-36, the largest improvements were seen in the dimension 'role limitations due to emotional problems' (change in mean scores of 6.1), followed by 'vitality' (5.7), and 'mental health' (5.1) (Supplementary Table e3).

\section{Psychometric validation of the AQoLS-Japan}

\section{Distributional properties}

Mean baseline dimension and total AQoLS-Japan scores were $<50$ on the 0 -to- 100 scale, reflecting the mild severity of disease in the sample (Table 2). Mean scores were slightly reduced with routine treatment (indicating improvement) between baseline and Week 2 and subsequently reduced further at the Month-3 visit (Table 2). Floor effects ( $>20 \%$ of the sample scoring at the minimum) were evident for the living conditions, control, and sleep dimensions, with the most substantial floor effects for the individual item of 'housing situation.' Ceiling effects were largely absent at all time points, and the AQoLS-Japan total score had no or only minimal floor or ceiling effects at any time point.

\section{Dimensional structure}

Inter-item and item-scale correlations at baseline, Week 2 , and Month 3 were moderate to strong. Very strong correlations $(>0.8)$, suggesting potential redundancy, were found among items in the activities (items $2-7,13,15$, and 25-26), control (items 28-32), and sleep (items 33 and 34) dimensions. The potential for redundancy among individual items in these domains was further indicated by the high corrected item-scale correlations $(>0.8)$ for a number of these items. Factor loadings from the CFA were all positive and statistically significant $(p<0.05)$. Two of the goodnessof-fit indices supported the adequacy of the AQoLS-Japan measurement model but one (the RMSEA) was higher than the recommended maximum value. Thus, although the CFA results generally supported the 7-factor structure of the AQoLS-Japan, there was evidence of interdependency among some items and factors (Supplementary Table e4).

\section{Reliability}

Cronbach's alpha coefficients for internal consistency at baseline ranged from 0.73 (living condition) to 0.97 (total score) (Table 3). The highest ICCs for test-retest reliability were found for the PGI-C stable subsample which was the primary indicator of stability in this evaluation. The total score and all dimensions except living conditions and sleep had reliability coefficients above the minimum recommended threshold of 0.70 . However, the lower bound of the $95 \%$ confidence interval fell below this threshold for all scores except the total, relationships, and self-esteem.

\section{Construct validity}

Convergent and divergent correlations (Fig. 1) confirmed moderate to strong negative $(|r| \geq 0.30)$ correlations with the SF-36 mental and role component summaries, but correlations were negligible to small for the physical component $(|r|<0.30$; [20]), and small to moderate for the EQ-5D visual analogue scale $(|r|=0.29-0.59)$. Small to moderate positive correlations were found with the CGI-S $(|r|=0.10-0.39)$ and alcohol consumption measures $(|r|=0.04-0.55)$, with higher positive correlations with the PGI-S $(|r|=0.28-0.58)$. Differences across known groups were statistically significant $(p<0.05)$ for most comparisons with PGI-S and CGI-S 
Table 2 AQoLS-Japan dimension and total descriptive statistics: baseline, Week-2, and Month-3 visits

\begin{tabular}{|c|c|c|c|}
\hline AQoLS dimension & Baseline & Week 2 & Month 3 \\
\hline \multicolumn{4}{|l|}{ Total } \\
\hline$n$ & 144 & 135 & 129 \\
\hline Mean (SD) & $30.4(22.5)$ & $26.6(22.2)$ & $16.8(18.4)$ \\
\hline Median (Q1, Q3) & $26.5(10.3,45.6)$ & $22.5(5.9,40.2)$ & $9.8(2.0,24.5)$ \\
\hline Minimum, maximum & $0.0,83.3$ & $0.0,91.2$ & $0.0,83.3$ \\
\hline$n(\%)$ scoring scale minimum & $6(4.0)$ & $5(3.4)$ & $14(10.6)$ \\
\hline$n(\%)$ scoring scale maximum & $0(0.0)$ & $0(0.0)$ & $0(0.0)$ \\
\hline$n(\%)$ missing & $6(4.0)$ & $10(6.9)$ & $3(2.3)$ \\
\hline \multicolumn{4}{|l|}{ Activities } \\
\hline$n$ & 147 & 139 & 130 \\
\hline Mean (SD) & $32.4(25.5)$ & $26.7(24.4)$ & $16.3(19.6)$ \\
\hline Median (Q1, Q3) & $26.7(10.0,53.3)$ & $20.0(3.3,43.3)$ & $10.0(0.0,20.0)$ \\
\hline Minimum, maximum & $0.0,96.7$ & $0.0,96.7$ & $0.0,86.7$ \\
\hline$n(\%)$ scoring scale minimum & $19(12.7)$ & $23(15.9)$ & $33(25.0)$ \\
\hline$n(\%)$ scoring scale maximum & $0(0.0)$ & $0(0.0)$ & $0(0.0)$ \\
\hline$n(\%)$ missing & $3(2.0)$ & $6(4.1)$ & $2(1.5)$ \\
\hline \multicolumn{4}{|l|}{ Relationships } \\
\hline$n$ & 148 & 142 & 132 \\
\hline Mean (SD) & $25.4(22.6)$ & $22.7(23.0)$ & $15.5(20.3)$ \\
\hline Median (Q1, Q3) & $22.2(5.6,38.9)$ & $16.7(5.6,33.3)$ & $5.6(0.0,27.8)$ \\
\hline Minimum, maximum & $0.0,83.3$ & $0.0,94.4$ & $0.0,94.4$ \\
\hline$n(\%)$ scoring scale minimum & $26(17.3)$ & $34(23.4)$ & $60(45.5)$ \\
\hline$n(\%)$ scoring scale maximum & $0(0.0)$ & $0(0.0)$ & $0(0.0)$ \\
\hline$n(\%)$ missing & $2(1.3)$ & $3(2.1)$ & $0(0.0)$ \\
\hline \multicolumn{4}{|l|}{ Living conditions } \\
\hline$n$ & 148 & 144 & 132 \\
\hline Mean (SD) & $21.4(22.6)$ & $16.7(19.7)$ & $11.2(17.2)$ \\
\hline Median (Q1, Q3) & $16.7(0.0,33.3)$ & $8.3(0.0,25.0)$ & $0.0(0.0,16.7)$ \\
\hline Minimum, maximum & $0.0,91.7$ & $0.0,83.3$ & $0.0,66.7$ \\
\hline$n(\%)$ scoring scale minimum & $43(28.7)$ & $56(38.6)$ & $75(56.8)$ \\
\hline$n(\%)$ scoring scale maximum & $0(0.0)$ & $0(0.0)$ & $0(0.0)$ \\
\hline$n(\%)$ missing & $2(1.3)$ & $1(0.7)$ & $0(0.0)$ \\
\hline \multicolumn{4}{|l|}{ Negative emotions } \\
\hline$n$ & 148 & 145 & 132 \\
\hline Mean (SD) & $44.9(31.0)$ & $42.8(30.7)$ & $27.7(29.3)$ \\
\hline Median (Q1, Q3) & $33.3(33.3,66.7)$ & $50.0(16.7,66.7)$ & $16.7(0.0,50.0)$ \\
\hline Minimum, maximum & $0.0,100.0$ & $0.0,100.0$ & $0.0,100.0$ \\
\hline$n(\%)$ scoring scale minimum & $27(18.0)$ & $29(20.0)$ & $54(40.9)$ \\
\hline$n(\%)$ scoring scale maximum & $13(8.7)$ & $9(6.2)$ & $4(3.0)$ \\
\hline$n(\%)$ missing & $2(1.3)$ & $0(0.0)$ & $0(0.0)$ \\
\hline \multicolumn{4}{|l|}{ Control } \\
\hline$n$ & 150 & 144 & 131 \\
\hline Mean (SD) & $31.6(29.2)$ & $26.0(28.9)$ & $13.8(22.2)$ \\
\hline Median (Q1, Q3) & $26.7(0.0,53.3)$ & $16.7(0.0,40.0)$ & $0.0(0.0,20.0)$ \\
\hline Minimum, maximum & $0.0,100.0$ & $0.0,100.0$ & $0.0,100.0$ \\
\hline$n(\%)$ scoring scale minimum & $42(28.0)$ & $48(33.1)$ & $75(56.8)$ \\
\hline$n(\%)$ scoring scale maximum & $5(3.3)$ & $3(2.1)$ & $1(0.8)$ \\
\hline$n(\%)$ missing & $0(0.0)$ & $1(0.7)$ & $1(0.8)$ \\
\hline \multicolumn{4}{|l|}{ Sleep } \\
\hline$n$ & 149 & 145 & 132 \\
\hline Mean (SD) & $39.3(33.3)$ & $35.3(33.4)$ & $25.1(29.9)$ \\
\hline
\end{tabular}


Table 2 (continued)

\begin{tabular}{llll}
\hline AQoLS dimension & Baseline & Week 2 & Month 3 \\
\hline Median (Q1, Q3) & $33.3(0.0,66.7)$ & $33.3(0.0,66.7)$ & $16.7(0.0,33.3)$ \\
Minimum, maximum & $0.0,100.0$ & $0.0,100.0$ & $0.0,100.0$ \\
$n(\%)$ scoring scale minimum & $42(28.0)$ & $43(29.7)$ & $60(45.5)$ \\
$n(\%)$ scoring scale maximum & $17(11.3)$ & $16(11.0)$ & $9(6.8)$ \\
$n(\%)$ missing & $1(0.7)$ & $0(0.0)$ & $0(0.0)$ \\
Self-esteem & & & \\
$n$ & 149 & 143 & 132 \\
Mean (SD) & $32.6(28.2)$ & $29.2(26.4)$ & $22.1(25.3)$ \\
Median (Q1, Q3) & $26.7(6.7,53.3)$ & $26.7(6.7,46.7)$ & $13.3(0.0,33.3)$ \\
Minimum, maximum & $0.0,100.0$ & $0.0,100.0$ & $0.0,100.0$ \\
$n(\%)$ scoring scale minimum & $23(15.3)$ & $32(22.1)$ & $46(34.8)$ \\
$n(\%)$ scoring scale maximum & $4(2.7)$ & $1(0.7)$ & $1(0.8)$ \\
$n(\%)$ missing & $1(0.7)$ & $2(1.4)$ & $0(0.0)$ \\
\hline
\end{tabular}

Table 3 Reliability of the AQoLS-Japan

\begin{tabular}{lllll}
\hline AQoLS-Japan dimension & $\begin{array}{l}\text { AQoLS-Japan Cronbach's alpha } \\
\text { coefficients for internal consist- } \\
\text { ency }\end{array}$ & $\begin{array}{l}\text { Intraclass correlation coefficients for } \\
\text { test-retest reliability over 2 weeks (95\% } \\
\text { CI) }\end{array}$ \\
\cline { 2 - 4 } & Baseline & Week 2 & Month 3 & \\
\hline Total & 0.97 & 0.97 & 0.97 & $0.80(0.70,0.88)$ \\
Activities & 0.92 & 0.93 & 0.91 & $0.74(0.60,0.83)$ \\
Relationships & 0.83 & 0.87 & 0.86 & $0.80(0.70,0.87)$ \\
Living conditions & 0.73 & 0.72 & 0.75 & $0.65(0.50,0.77)$ \\
Negative emotions & 0.83 & 0.82 & 0.81 & $0.74(0.62,0.83)$ \\
Control & 0.92 & 0.94 & 0.93 & $0.70(0.56,0.80)$ \\
Sleep & 0.93 & 0.93 & 0.94 & $0.65(0.49,0.76)$ \\
Self-esteem & 0.91 & 0.91 & 0.92 & $0.82(0.72,0.88)$ \\
\hline
\end{tabular}

Intraclass correlation coefficients are shown for the stable subsample (based on PGI-C, $n=74$ ) at baseline, with the only non-significant exception being the association between CGI-S and living conditions. There was also a clear pattern of higher scores (indicating worse HRQoL) with increasing levels of drinking-days consumption across most of the AQoLS-Japan dimensions. Baseline differences between the low ( $<5$ HDDs) and high HDD $(\geq 14$ HDDs) groups were statistically significant $(p<0.05)$ for most AQoLS-Japan dimensions except relationships and living conditions. Similarly, statistically significant $(p<0.05)$ baseline differences were observed between patients with low and high or very high DRL on all AQoLS dimensions except living conditions.

\section{Responsiveness}

In general, the pattern of associations with the SF-36 and consumption measures was similar to that observed with the cross-sectional scores in the convergent and divergent construct validity assessments, with moderate correlations between AQoLS-Japan change scores and changes on the mental domains of the SF-36 but weaker correlations with changes on the physical domains of the SF-36. Changes in AQoLS-Japan scores were generally more highly associated with change on PGI-C than CGI-I. Evaluation of within-group change demonstrated that the AQoLS-Japan total and all domains were consistently able to demonstrate statistically significant improvement ( $p<0.001$ in all cases) in HR-QoL over time. Mean \pm SD change on the AQoLSJapan total was $-13.7 \pm 20.9$ for the overall sample, which increased to between $-16.7 \pm 21.7$ and $-24.9 \pm 18.9$ for patients who were classified as having improved on the global impression of change assessments and alcohol consumption measures, respectively. Estimates for effect size and standardized response means for the AQoLS total and dimension scores were small to moderate (i.e., $\geq 0.20$ to $<0.80$ ) for change in the overall sample and moderate (i.e., $\geq 0.50$ to $<0.80$ ) for change associated with a defined improvement based on PGI-C and CGI-I. For most of the AQoLS dimensions (except for sleep and self-esteem), the estimates for effect size and standardized response means 
(a)

Measure

\section{SF-36 MCS}

SF-36 PCS

SF-36 RCS

SF-36 bodily pain

SF-36 general health

SF-36 mental health

SF-36 physical functioning

SF-36 role emotiona!

SF-36 role physical

SF-36 social functioning

SF-36 vitality

EQ-5D VAS

EQ-5D Utility Index

PGI-S

CGI-S

No. of Drinking days

HDDs

TAC

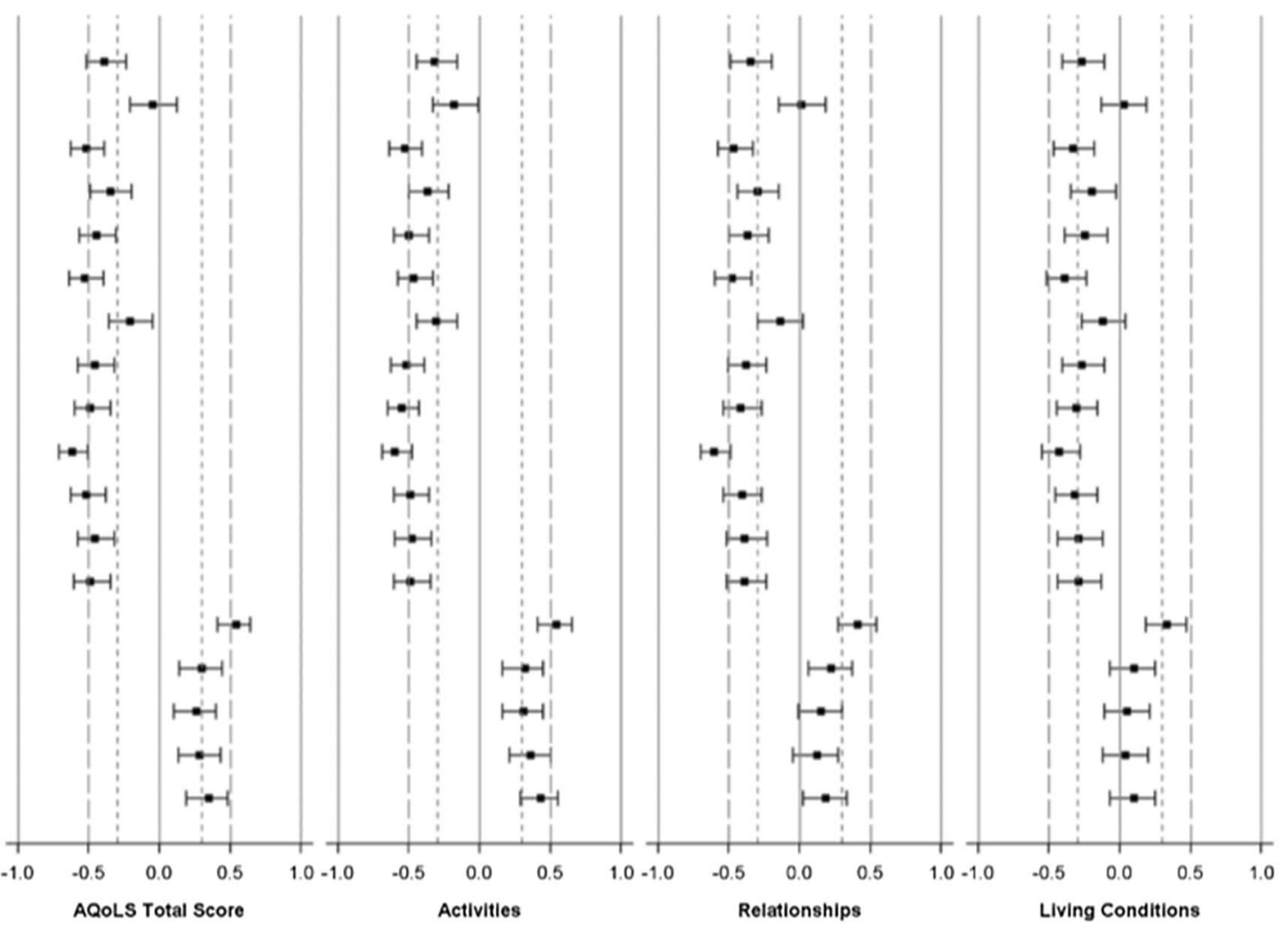

(b)

Measure
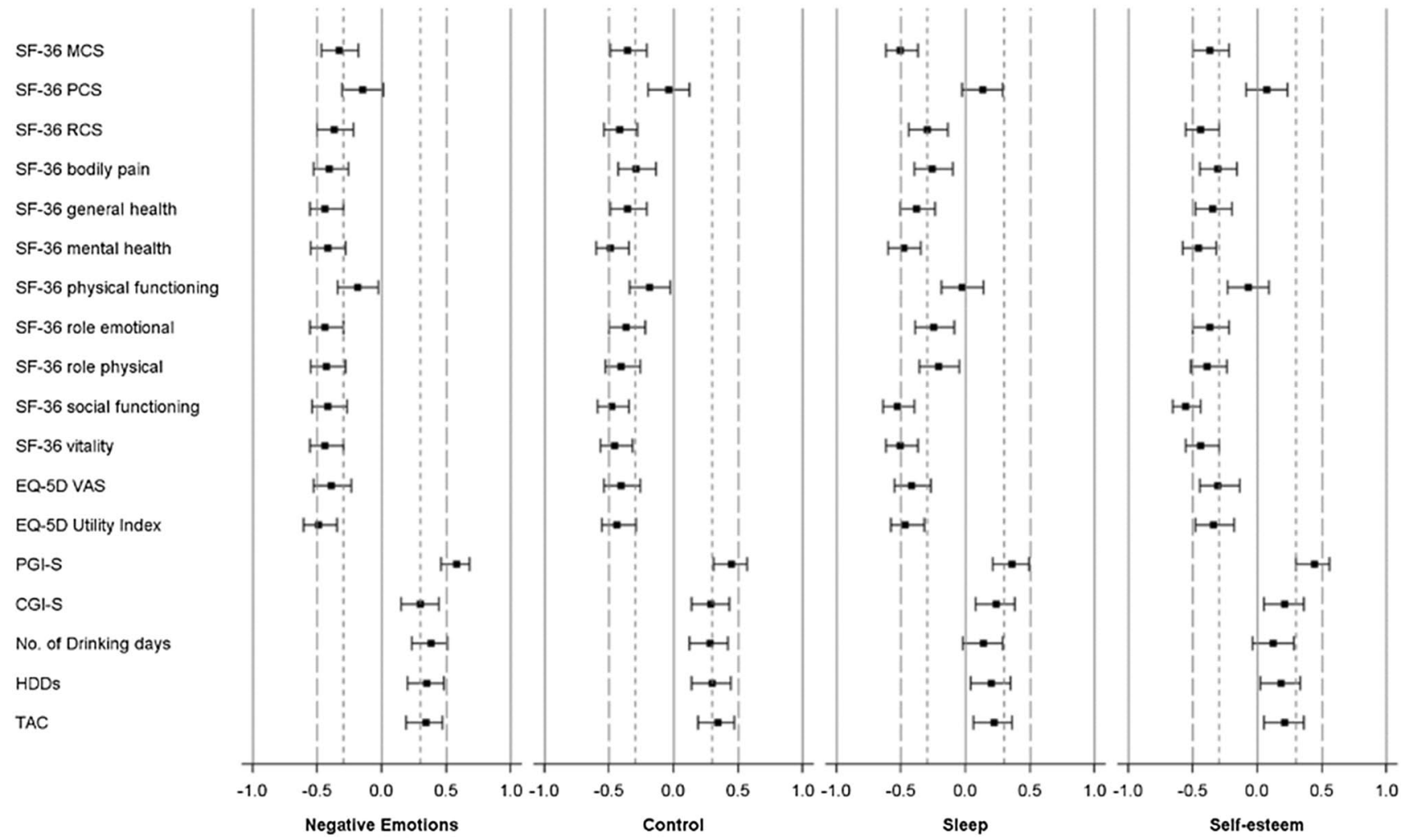
४Fig. 1 Construct validity: correlations between scores on the AQoLS and other measures at baseline. a AQoLS Total, activities, relationships, and living conditions dimensions. b AQoLS negative emotions, control, sleep, and self-esteem dimensions. AQoLS Alcohol Quality of Life Scale, $C G I-S$ clinical global impression-severity, $H D D$ heavy drinking day, $M C S$ mental component summary, $P C S$ physical component summary, $P G I-S$ patient global impression-severity, $T A C$ total alcohol consumption

were large (i.e., $\geq 0.80$ ) for change associated with a defined improvement as measured by HDDs and TAC (Table 4).

\section{Interpretation of change}

Estimates for the MCID of group-level change on AQoLSJapan total scores were -18.2 based on PGI-S, -13.2 based on PGI-C, and -13.5 based on DRL (Table 5). For grouplevel differences, the MCID of group-level differences were -15.7 based on PGI-S, -9.7 based on PGI-C, and -2.4 based on DRL.

Moderate improvements (defined as PGI-S improvement of 2, PGI-C score of 2, or a DRL response) corresponded to mean changes in AQoLS-Japan total scores of -24.7 based on PGI-S, -17.6 based on PGI-C, and -21.5 based on DRL. Based on the sensitivity and specificity of ROC in determining a responder definition, an appropriate responder definition on the AQoLS-Japan total could be considered as an improvement of between 8.8 and 14.7 points. Cumulative distribution function plots showed that $50 \%$ of patients who had a PGI-C score of 2 (much better) achieved an improvement of between 15 and 20 points on the AQoLS-Japan total. For change in DRL, $50 \%$ of patients whose drinking decreased by one DRL achieved an improvement of 10 and 15 points on the AQoLS-Japan total, whereas $50 \%$ of patients whose drinking decreased by two or three DRLs achieved an improvement of approximately 30 points on the AQoLS-Japan total.

\section{Discussion}

The primary aim of this study was to evaluate the recently translated AQoLS-Japan. We show that the AQoLS-Japan is a reliable and valid measure of HR-QoL that is able to demonstrate benefits associated with treatment of patients with AUD.

Although the results suggested that the individual dimensions are consistent and that the dimensional structure holds, a number of dimensions in the Japanese version had Cronbach's alpha coefficients above the recommended optimal range (0.70-0.90), suggesting redundancy among the items of the AQoLS-Japan and the model fit was not as strong as for the UK and French versions. For example, inter-item correlations showed potential redundancy for the items of activities and control. Such findings confirm those of the previous validation study of the measure in the UK and France, which also highlighted the potential to reduce the number of items and/or to simplify the dimensional structure [11]. Although any attempts to remove items would need to be carefully considered in relation to the face and content validity of the overall measure, it is considered that simplification of the AQoLS-Japan could provide a more practical and efficient assessment of HR-QoL in the context of clinical trials and routine clinical practice.

Our results demonstrate good support for the construct validity of the Japanese AQoLS. Correlations with the SF-36, EQ-5D, as well as the patient- and clinician-reported global assessments were as expected, and the pattern of correlations was similar to the pattern obtained with the UK and French versions of the AQoLS [11]. As with the earlier validation study (English/French versions) [11], AQoLSJapan total scores were more strongly associated with the SF-36 mental component scores than the physical component scores. Thus, our data support the wider literature showing that alcohol dependence impacts mental health more than the physical components of HR-QoL [26, 27]. In our study, we also sought to understand known-groups validity of the scale (not previously reported for the English and French versions). The AQoLS-Japan was consistently able to discriminate between groups based on patient- and clinician-reported severity of disease (PGI-S and CGI-S) and consumption measures (drinking days, HDDs, and DRL). Overall, the total, activities, and control dimensions of the AQoLS had the greatest discriminating ability across levels of consumption. The least discriminatory AQoLS dimensions were relationships and living conditions, which may reflect the Japanese tolerance to excessive alcohol consumption behavior [5, 6].

A limitation of this study is that we did not test whether the AQoLS-Japan is more sensitive than generic measures of HR-QoL (SF-36 and EQ-5D). However, we demonstrated the strength of responsiveness among patients with defined improvements in the global impression assessments and alcohol consumption measures and this was shown by the moderate to strong effect-size estimates for change associated with defined improvements in the global impression assessments and alcohol consumption measures. Ascertaining the MCID can help assess whether a statistically significant treatment effect in a clinical trial is sufficiently large enough to be interpreted as clinically significant and could help personalize treatment in clinical practice. Overall, for the AQoLS-Japan total score, a MCID of between 10 and 15 points is recommended for interpreting group-level differences or change and a responder definition of between 15 and 25 points is recommended for evaluating individuallevel change. It is currently recommended that estimation of MCID for any given scale should be based on multiple 
Table 4 Ability to detect change-effect-size estimates and standardized response means

\begin{tabular}{|c|c|c|c|c|}
\hline AQoLS dimension & $\begin{array}{l}\text { Change in AQoLS } \\
\text { Mean (SD), } n\end{array}$ & $\begin{array}{l}\text { t-Statistic } \\
\text { ( } p \text { value) }\end{array}$ & $\begin{array}{l}\text { Effect-size } \\
\text { estimate }\end{array}$ & $\begin{array}{l}\text { Standard- } \\
\text { ized response } \\
\text { mean }\end{array}$ \\
\hline \multicolumn{5}{|l|}{ Whole sample } \\
\hline Total score & $-13.7(20.9), 124$ & $7.33(<0.001)$ & -0.61 & -0.66 \\
\hline Activities & $-15.9(24.7), 127$ & $7.23(<0.001)$ & -0.62 & -0.64 \\
\hline Relationships & $-10.1(22.4), 131$ & $5.17(<0.001)$ & -0.45 & -0.45 \\
\hline Living conditions & $-10.8(23.8), 130$ & $5.19(<0.001)$ & -0.48 & -0.45 \\
\hline Negative emotions & $-16.9(29.5), 130$ & $6.54(<0.001)$ & -0.55 & -0.57 \\
\hline Control & $-16.7(28.3), 131$ & $6.78(<0.001)$ & -0.57 & -0.59 \\
\hline Sleep & $-12.5(33.7), 131$ & $4.24(<0.001)$ & -0.37 & -0.37 \\
\hline Self-esteem & $-10.4(25.3), 131$ & $4.72(<0.001)$ & -0.37 & -0.41 \\
\hline \multicolumn{5}{|c|}{ PGI-C improved (score $\leq 3$ at Month 3 ) } \\
\hline Total score & -16.7 (21.7), 99 & $7.66(<0.001)$ & -0.72 & -0.77 \\
\hline Activities & $-18.8(25.7), 102$ & $7.36(<0.001)$ & -0.74 & -0.73 \\
\hline Relationships & $-13.4(23.0), 104$ & $5.95(<0.001)$ & -0.58 & -0.58 \\
\hline Living conditions & $-14.9(24.0), 103$ & $6.31(<0.001)$ & -0.61 & -0.62 \\
\hline Negative emotions & $-18.8(30.6), 103$ & $6.23(<0.001)$ & -0.59 & -0.61 \\
\hline Control & $-21.6(28.2), 104$ & $7.80(<0.001)$ & -0.74 & -0.76 \\
\hline Sleep & $-16.2(33.0), 104$ & $5.00(<0.001)$ & -0.49 & -0.49 \\
\hline Self-esteem & $-13.1(26.5), 104$ & $5.03(<0.001)$ & -0.45 & -0.49 \\
\hline \multicolumn{5}{|c|}{ CGI-I improved (score $\leq 3$ at Month 3 ) } \\
\hline Total score & -17.5 (20.2), 75 & $7.50(<0.001)$ & -0.79 & -0.87 \\
\hline Activities & $-21.4(25.9), 76$ & $7.21(<0.001)$ & -0.84 & -0.83 \\
\hline Relationships & $-13.6(21.0), 77$ & $5.69(<0.001)$ & -0.59 & -0.65 \\
\hline Living conditions & $-14.5(21.2), 76$ & $5.94(<0.001)$ & -0.70 & -0.68 \\
\hline Negative emotions & $-21.4(29.0), 77$ & $6.49(<0.001)$ & -0.69 & -0.74 \\
\hline Control & $-23.2(27.6), 78$ & $7.40(<0.001)$ & -0.81 & -0.84 \\
\hline Sleep & $-13.0(32.5), 77$ & $3.50(<0.001)$ & -0.38 & -0.40 \\
\hline Self-esteem & $-13.0(25.5), 77$ & $4.46(<0.001)$ & -0.47 & -0.51 \\
\hline \multicolumn{5}{|c|}{ HDD improved (0-4 HDDs at 3 months, excluding patients who had $\leq 4$ HDDs at baseline) } \\
\hline Total score & $-24.9(18.9), 37$ & $8.00(<0.001)$ & -1.55 & -1.31 \\
\hline Activities & $-29.8(24.7), 39$ & $7.54(<0.001)$ & -1.52 & -1.21 \\
\hline Relationships & $-17.1(22.1), 40$ & $4.89(<0.001)$ & -0.80 & -0.77 \\
\hline Living conditions & -17.5 (21.3), 39 & $5.14(<0.001)$ & -0.90 & -0.82 \\
\hline Negative emotions & $-30.8(34.3), 39$ & $5.60(<0.001)$ & -1.28 & -0.90 \\
\hline Control & $-35.4(23.8), 41$ & $9.53(<0.001)$ & -1.65 & -1.49 \\
\hline Sleep & $-26.7(35.8), 40$ & $4.72(<0.001)$ & -0.79 & -0.75 \\
\hline Self-esteem & $-15.8(25.4), 40$ & $3.94(<0.001)$ & -0.61 & -0.62 \\
\hline \multicolumn{5}{|c|}{ TAC improved (50\% reduction in TAC between baseline and 3 months) } \\
\hline Total score & $-21.6(19.2), 58$ & $8.59(<0.001)$ & -1.20 & -1.13 \\
\hline Activities & $-26.1(24.3), 60$ & $8.34(<0.001)$ & -1.24 & -1.08 \\
\hline Relationships & $-15.6(22.1), 61$ & $5.50(<0.001)$ & -0.72 & -0.70 \\
\hline Living conditions & $-16.5(20.5), 60$ & $6.25(<0.001)$ & -0.85 & -0.81 \\
\hline Negative emotions & $-27.5(31.0), 60$ & $6.88(<0.001)$ & -1.07 & -0.89 \\
\hline Control & $-31.1(27.0), 62$ & $9.05(<0.001)$ & -1.27 & -1.15 \\
\hline Sleep & $-17.8(37.9), 61$ & $3.66(<0.001)$ & -0.52 & -0.47 \\
\hline Self-esteem & $-14.3(24.6), 61$ & $4.55(<0.001)$ & -0.53 & -0.58 \\
\hline
\end{tabular}

approaches and triangulation of methods [23]. In our analyses, the MCID estimate for a group-level difference arising from the DRL anchor was considerably lower than the other estimates (PGI-C and PGI-S). This may be because each DRL includes a broad range of consumption, making it possible for patients to have a meaningful change in HR-QoL 
Table 5 Mean scores and correlations for AQoLS Total change from baseline scores at 3 months for potential external anchors

\begin{tabular}{|c|c|c|c|c|}
\hline Anchor & Change level & $n$ & $\begin{array}{l}\text { Mean (SD) change } \\
\text { on AQoLS total }\end{array}$ & $\begin{array}{l}\text { Correlation }(95 \% \mathrm{CI}) \\
\text { with change in AQoLS } \\
\text { total }\end{array}$ \\
\hline \multirow[t]{5}{*}{ PGI-S change between baseline and 3 months } & Improved 3 or 4 points & 10 & $-37.4(23.0)$ & \multirow[t]{5}{*}{$0.42(0.26,0.56)$} \\
\hline & Improved 2 points & 17 & $-24.7(22.9)$ & \\
\hline & Improved 1 point & 29 & $-18.2(16.8)$ & \\
\hline & No change & 46 & $-2.5(17.6)$ & \\
\hline & Worsened 1 to 3 points & 20 & $-10.8(14.5)$ & \\
\hline \multirow[t]{5}{*}{ PGI-C score at 3 months } & Very much better & 36 & $-18.7(20.6)$ & \multirow[t]{5}{*}{$0.28(0.11,0.44)$} \\
\hline & Much better & 34 & $-17.6(17.9)$ & \\
\hline & A little better & 29 & $-13.2(26.8)$ & \\
\hline & No change & 18 & $-3.5(7.9)$ & \\
\hline & A little or much worse & 6 & $4.2(18.9)$ & \\
\hline \multirow[t]{5}{*}{ CGI-S change between baseline and 3 months } & Improved 3 to 5 points & 10 & $-24.3(23.9)$ & \multirow[t]{5}{*}{$0.22(0.05,0.38)$} \\
\hline & Improved 2 points & 15 & $-15.1(19.6)$ & \\
\hline & Improved 1 point & 23 & $-19.1(19.7)$ & \\
\hline & No change & 64 & $-12.2(20.9)$ & \\
\hline & Worsened 1 to 3 points & 12 & $-1.0(16.8)$ & \\
\hline \multirow[t]{5}{*}{ CGI-I score at 3 months } & Very much improved & 25 & $-16.8(18.4)$ & \multirow[t]{5}{*}{$0.20(0.03,0.37)$} \\
\hline & Much improved & 26 & $-16.6(21.9)$ & \\
\hline & Minimally improved & 24 & $-19.2(20.9)$ & \\
\hline & No change & 36 & $-9.8(21.5)$ & \\
\hline & Minimally worse to very much worse & 13 & $-2.8(18.3)$ & \\
\hline \multirow[t]{4}{*}{ DRL change between baseline and 3 months } & Decreased 2 or 3 levels & 16 & $-32.1(21.0)$ & \multirow[t]{4}{*}{$0.34(0.17,0.48)$} \\
\hline & Decreased 1 level & 15 & $-13.5(17.1)$ & \\
\hline & No change & 89 & $-11.1(19.6)$ & \\
\hline & Increased 1 to 3 levels & 4 & $-0.5(27.0)$ & \\
\hline \multirow[t]{2}{*}{ DRL response } & Response & 54 & $-21.5(19.8)$ & \multirow[t]{2}{*}{$0.34(0.17,0.48)$} \\
\hline & $\begin{array}{l}\text { Non-response (change that did not } \\
\text { meet the criteria for DRL response) }\end{array}$ & 30 & $-8.2(20.5)$ & \\
\hline
\end{tabular}

DRL response was defined as change in DRL between baseline and 3 months: from very high to medium or below, from high or medium to low or below, or from low to no alcohol consumption

without an associated change in risk level. Thus, the mean score difference between a reduction of one DRL and no change (i.e., 2.4 points) may be an underestimate of the true MCID for the AQoLS-Japan.

Strengths of the study include its prospective, longitudinal design and the high number of treated patients; the study was focused on clinical practice and included physicians from across Japan. However, this naturalistic design was reflected in the relatively high percentage of patients with low to medium DRL and we may have seen less redundancy of items if we had included more patients with more severe alcohol dependence. The inclusion of low risk DRL also restricted our ability to assess response by reductions in DRL (since, by definition, response in patients at low DRL could be assessed only when they reached zero alcohol consumption after treatment). In addition, most patients had abstinence as their treatment goal, whereas there is a move in the field towards reduced drinking as an alternative goal that is often more acceptable to patients [6, 28]. Such limitations should be considered when using our data or the AQoLS-Japan for designing a clinical trial where the focus of treatment may be reduction of alcohol (vs. abstinence) in patients at higher DRL.

\section{Conclusion}

This psychometric evaluation indicates that the AQoLSJapan is a reliable and valid measure of HR-QoL that can demonstrate benefits associated with treatment. Our data indicate a shorter version could be developed, which may make it easier to use in clinical research and practice. Our comprehensive approach to characterizing the scale psychometrics will aid future clinical trial design in Japanese subjects with AUD, who carry significant health burdens. 
Acknowledgements This work was supported by H. Lundbeck A/S. The authors thank all the investigators and patients who participated in this trial and would like to acknowledge the help from Johnny Wong (Lundbeck Singapore Pte Ltd) in reviewing the statistics and editorial assistance of Anita Chadha-Patel (ACP Clinical Communications Ltd, funded by $\mathrm{H}$. Lundbeck A/S) who assisted with table preparation, referencing, and final styling under full direction of the authors.

Funding Funding for this study was provided by Lundbeck SAS.

\section{Compliance with ethical standards}

Conflict of interest Susumu Higuchi has received personal fees, grants, and other fees from Otsuka Pharmaceutical, as wells as grants and other fees from Lundbeck Japan during the conduct of the study as well as personal fees from Nippon Shinyaku, MSD, Yoshitomi Pharmaceutical, Jansen Pharma, Eli-Lilly Japan, Mochida Pharmaceutical, and Meiji-Seika Pharma and other fees from Nippon Shinyaku, Eisai, and Meiji-Seika Pharma, outside the submitted work. Yoshiya Moriguchi is employed by Lundbeck Japan K.K. (Tokyo, Japan) and Kristin Hui Xian Tan was employed by Lundbeck Singapore Pte Ltd at the time of study.

Research involving human participants All procedures performed in studies involving human participants were in accordance with the ethical standards of the local and institutional research committees and with the 1964 Helsinki declaration and its later amendments. Ethical approval was granted through the Ethics committee of Clinical Research Promotion Network Japan (CRPN-J).

Informed consent All patients provided written informed consent to participate in the trial.

Open Access This article is distributed under the terms of the Creative Commons Attribution 4.0 International License (http://creativeco mmons.org/licenses/by/4.0/), which permits unrestricted use, distribution, and reproduction in any medium, provided you give appropriate credit to the original author(s) and the source, provide a link to the Creative Commons license, and indicate if changes were made.

\section{References}

1. Lim, S. S., Vos, T., Flaxman, A. D., Danaei, G., Shibuya, K., Adair-Rohani, H., et al. (2010). A comparative risk assessment of burden of disease and injury attributable to 67 risk factors and risk factor clusters in 21 regions, 1990-2010: A systematic analysis for the Global Burden of Disease Study 2010. Lancet, 380, 2224-2260.

2. WHO. Global Status Report on Alcohol \& Health, 2018.

3. Kumagai, Y., Morioka, I., Yura, S., \& Hisatomi, N. (2004). Actual conditions of heavy drinkers in a community and measures against alcoholic problems]. Nihon Arukoru Yakubutsu Igakkai Zasshi, 39, 180-188.

4. WhitePaperonAlcohol. White Paper on Alcohol. 2011. Japan (a simplified version available only in Japanese): Japanese Society of Alcohol-related Problems, Japanese Medical Society of Alcohol and Drug Studies, Japanese Society of Biological Psychiatry (eds).

5. Shimizu, S., Kim, D. S., \& Hirota, M. (2004). Drinking practice and alcohol-related problems: The national representative sample survey for Healthy Japan 21. Nihon Arukoru Yakubutsu Igakkai Zasshi, 39, 189-206.

6. Taguchi, Y., Takei, Y., Sasai, R., \& Murteira, S. (2014). Awareness and treatment of alcohol dependence in Japan: Results from internet-based surveys in persons, family, physicians and society. Alcohol and Alcoholism, 49, 439-446.

7. Laudet, A. B. (2011). The case for considering quality of life in addiction research and clinical practice. Addiction Science \& Clinical Practice, 6, 44-55.

8. Luquiens, A., Reynaud, M., Falissard, B., \& Aubin, H. J. (2012). Quality of life among alcohol-dependent patients: How satisfactory are the available instruments? A systematic review. Drug and Alcohol Dependence, 125, 192-202.

9. Whalley, D., Crawford, S. R., Laramee, P., Higuchi, S., Hao, W., Kim, S. G., et al. (2014). Cultural adaptation of the Alcohol Quality of Life Scale for use in Japan, China, and Korea (Abstract). Value Health, 17(7), A462.

10. Luquiens, A., Whalley, D., Crawford, S. R., Laramee, P., Doward, L., Price, M., et al. (2015). Development of the Alcohol Quality of Life Scale (AQoLS): A new patient-reported outcome measure to assess health-related quality of life in alcohol use disorder. Quality of Life Research, 24, 1471-1481.

11. Luquiens, A., Whalley, D., Laramee, P., Falissard, B., Kostogianni, N., Rehm, J., et al. (2016). Validation of a new patientreported outcome instrument of health-related quality of life specific to patients with alcohol use disorder: The Alcohol Quality of Life Scale (AQoLS). Quality of Life Research, 25, $1549-1560$

12. EuroQol Group. (1990). EuroQol-a new facility for the measurement of health-related quality of life. Health Policy, 16, 199-208.

13. Ware, J. E., \& Sherbourne, C. D. (1992). The MOS 36-item shortform health survey (SF-36). I. Conceptual framework and item selection. Medical Care, 30, 473-483.

14. Sobell, L. C., \& Sobell, M. B. (1992). Timeline follow-back: A technique for assessing self-reported ethanol consumption. In R. Z. Litten \& J. P. Allen (Eds.), Measuring alcohol consumption: Psychosocial and biological methods (pp. 41-72). Totowa: Humana Press.

15. Guy W (ed). ECDEU Assessment Manual for Psychopharmacology. Rockville, MD: US Department of Heath,Education, and Welfare Public Health Service Alcohol, Drug Abuse, and Mental Health Administration, 1976

16. Bujang, M. A., \& Baharum, N. (2017). A simplified guide to determination of sample size requirements for estimating the value of intraclass correlation coefficient: A review. Archives of Orofacial Sciences, 12, 1-11.

17. Mann, K., Bladstrom, A., Torup, L., Gual, A., \& van den Brink, W. (2013). Extending the treatment options in alcohol dependence: A randomized controlled study of as-needed nalmefene. Biological Psychiatry, 73, 706-713.

18. Gual, A., He, Y., Torup, L., van den Brink, W., Mann, K., \& Group ES. (2013). A randomised, double-blind, placebo-controlled, efficacy study of nalmefene, as-needed use, in patients with alcohol dependence. European Neuropsychopharmacology, 23, 1432-1442.

19. Streiner, D. L., \& Norman, G. R. (1995). Health measurement scales: A practical guide to their development and use (2nd ed.). New York: Oxford University Press.

20. Cohen, J. (1992). A power primer. Psychological Bulletin, 112(1), 155-159.

21. Rehm, J., Baliunas, D., Borges, G. L., Graham, K., Irving, H., Kehoe, T., et al. (2010). The relation between different dimensions of alcohol consumption and burden of disease: An overview. Addiction, 105, 817-843.

22. Cohen, J. (1988). Statistical power analysis for the behavioral sciences (2nd ed.). Hillsdale: Lawrence Erlbaum Associates. 
23. Revicki, D., Hays, R. D., Cella, D., \& Sloan, J. (2008). Recommended methods for determining responsiveness and minimally important differences for patient-reported outcomes. Journal of Clinical Epidemiology, 61, 102-109.

24. Coon, C. D., \& Cappelleri, J. C. (2016). Interpreting change in scores on patient-reported outcome instruments. Therapeutic Innovation \& Regulatory Science, 50(1), 22-29.

25. McLeod, L. D., Coon, C. D., Martin, S. A., Fehnel, S. E., \& Hays, R. D. (2011). Interpreting patient-reported outcome results: US FDA guidance and emerging methods. Expert Review of Pharmacoeconomics \& Outcomes Research, 11(2), 163-169.

26. Malet, L., Llorca, P. M., Beringuier, B., Lehert, P., \& Falissard, B. (2006). AlQoL 9 for measuring quality of life in alcohol dependence. Alcohol and Alcoholism, 41, 181-187.
27. Daeppen, J. B., Faouzi, M., Sanchez, N., Rahhali, N., Bineau, S., \& Bertholet, N. (2014). Quality of life depends on the drinking pattern in alcohol-dependent patients. Alcohol and Alcoholism, 49(4), 457-465.

28. Ambrogne, J. A. (2002). Reduced-risk drinking as a treatment goal: What clinicians need to know. Journal of Substance Abuse Treatment, 22, 45-53.

Publisher's Note Springer Nature remains neutral with regard to jurisdictional claims in published maps and institutional affiliations. 